\title{
Feasibility, safety, and outcomes of a single-step endoscopic ultrasonography-guided drainage of pancreatic fluid collections without fluoroscopy using a novel electrocautery- enhanced lumen-apposing, self-expanding metal stent
}

\author{
Joseph Yoo, Linda Yan, Raza Hasan, Saana Somalya, Jose Nieto ${ }^{1}$, Ali A. Siddiqui \\ Division of Gastroenterology and Hepatology, Thomas Jefferson University, Philadelphia, PA, ${ }^{1}$ Division of Gastroenterology, \\ Borland Groover Clinic, Jacksonville, FL, USA
}

\begin{abstract}
Background and Objectives: There are currently limited data available regarding the safety of endoscopic ultrasound (EUS)-guided drainage of pancreatic fluid collections (PFCs) using the lumen-apposing metal stent without fluoroscopic guidance. This study aims to evaluate clinical outcomes and safety of EUS-guided drainage of PFC using the electrocautery-enhanced lumen-apposing metal stents (EC-LAMSs) without fluoroscopic guidance. Methods: We conducted a retrospective study on patients with symptomatic PFC who underwent EUS-guided drainage using EC-LAMS without fluoroscopy. All patients were followed clinically until resolution of their PFC. Technical success (successful placement of EC-LAMS), number of patients who achieved complete resolution of PFC without additional intervention and adverse events were noted. Results: We evaluated 25 patients, including three with pancreatic pseudocysts and 22 with walled-off necrosis (WON). The etiology of the patient's pancreatitis was gallstones (42\%), alcohol (27\%), and other causes (31\%). The mean cyst size was $82 \mathrm{~mm}$ (range, 60-170 mm). The indications for endoscopic drainage were abdominal pain, infected WON, or gastric outlet obstruction. Technical success with placement of the EC-LAMS was achieved in all 25 patients. There were no procedure-related complications. The mean patient follow-up was 7.8 months. PFCs resolved in $24(96 \%)$ patients; the one failure was in a patient with WON. Stent occlusion was seen in one patient. There was a spontaneous migration of one stent into the enteral lumen after resolution of WONs. The EC-LAMS were successfully removed using a snare in all the remaining patients. The median number of endoscopy sessions to achieve PFCs resolution was 2 (range, 2-6). Conclusions: Single-step EUS-guided drainage of PFCs without fluoroscopic guidance using the novel EC-LAMS is a safe and effective endoscopic technique for drainage of PFCs with excellent technical and clinical success rates and no complications. Due to its ease of use, EC-LAMS may simplify and streamline EUS-guided management of PFC and help in its widespread adoption as an alternative to surgery.
\end{abstract}

Key words: Endosonography, pancreatic fluid collections, stents

\begin{tabular}{|l|l|}
\hline \multicolumn{2}{|c|}{ Access this article online } \\
\hline Quick Response Code: & Website: \\
\hline & www.eusjournal.com \\
\hline & \\
\hline Dol:
\end{tabular}

This is an open access article distributed under the terms of the Creative Commons Attribution-NonCommercial-ShareAlike 3.0 License, which allows others to remix, tweak, and build upon the work non-commercially, as long as the author is credited and the new creations are licensed under the identical terms.

For reprints contact: reprints@medknow.com

How to cite this article: Yoo J, Yan L, Hasan R, Somalya S, Nieto J, Siddiqui AA. Feasibility, safety, and outcomes of a single-step endoscopic ultrasonography-guided drainage of pancreatic fluid collections without fluoroscopy using a novel electrocautery-enhanced lumen-apposing, self-expanding metal stent. Endosc Ultrasound 2017;6:131-5.

\section{Address for correspondence}

Dr. Ali A. Siddiqui, Division of Gastroenterology, Thomas Jefferson University, Philadelphia, PA, USA. E-mail: ali.siddiqui@jefferson.edu Received: 2016-05-16; Accepted: 2016-10-26 
Yoo, et al.: Electrocautery-enhanced lumen-apposing stent for pancreatic fluid collections without fluoroscopy

\section{INTRODUCTION}

Pancreatic fluid collections (PFCs) develop in the setting of damage to the major pancreatic duct and/or peripheral ducts as a complication of acute or chronic pancreatitis, trauma, iatrogenic causes (i.e., surgery), or in patients with disconnected duct syndrome. ${ }^{[1-3]}$ PFCs include pancreatic pseudocysts (PP) and walled-off necrosis (WON). ${ }^{[1]}$ The majority of PFCs are asymptomatic and will resolve spontaneously. ${ }^{[4]}$ However, if a PFC persists, therapeutic intervention is indicated when complications develop, such as infection, compression of neighboring large vessels, or obstruction of the duodenum, stomach, or common bile duct. ${ }^{[5]}$ Intervention is also indicated when PFCs cause symptoms such as nausea, vomiting, abdominal pain, or early satiety. ${ }^{[4]}$

The current therapeutic interventions available for PFCs include surgical, percutaneous, and endoscopic drainage. ${ }^{[6,7]}$ Studies have demonstrated that the success rate of endoscopic intervention is comparable to that of percutaneous and surgical intervention. Endoscopic intervention has decreased morbidity, length of hospital stay, and cost when compared to percutaneous or surgical intervention. ${ }^{[8,9]}$ The surgical approach is associated with a high morbidity and mortality, whereas the percutaneous approach carries the risk of fistula formation, cyst recurrence, and infections. In the last decade, endoscopic-guided drainage of PFCs through placement of transmural stents has become the procedure of choice and is considered first-line therapy. ${ }^{[10,11]}$ The clinical success of endoscopic-guided PFC drainage is related to the type of collection, with a success rate $>90 \%$ for PPs, and a slightly lower success rate of $50 \%-65 \%$ for $\mathrm{WON}$, given the increased risk of stent occlusion in the presence of necrotic contents. ${ }^{[9]}$

Recently, a novel "saddle-shaped" lumen-apposing fully covered self-expanding metal stent (LAMS) has been used and shown to be safe and effective for endoscopic transmural drainage of PFCs with technical success rates of $89 \%-100 \%$ and clinical success rates of $88 \%-100 \%{ }^{[12-14]}$

The latest development in therapeutic options is the LAMS with an electrocautery-enhanced delivery system (EC-LAMS). EC-LAMS may enable clinicians to perform endoscopic-guided drainage of PFCs in a faster and safer manner by decreasing the number of steps needed. In addition, while endoscopic-guided drainage of PFCs has typically utilized fluoroscopy to optimize visualization and access into the PFCs, the EC-LAMS system has the potential to be done safely and effectively without fluoroscopic guidance. While the use of LAMS under fluoroscopic guidance has already been shown to be promising, ${ }^{[14]}$ the objective of this retrospective study was to demonstrate the safety and clinical outcomes of endoscopic ultrasound (EUS)-guided drainage of PFCs using EC-LAMS without fluoroscopic guidance.

\section{METHODS}

This was a multi-center retrospective study conducted at two tertiary care centers and approved by the Institutional Review Boards at both centers.

Patients who underwent EUS-guided drainage of PFCs using the EC-LAMS without fluoroscopic guidance were identified from the endoscopy database at Thomas Jefferson University Hospital and Borland-Groover Clinic. WON was identified as a mature, encapsulated collection of pancreatic and/or peripancreatic necrotic tissue enclosed in an enhancing wall of reactive tissue. PPs were defined as encapsulated collections of fluid within a well-defined inflammatory wall usually outside the pancreas with minimal or no necrosis (as per the Revised Atlanta Classification). ${ }^{[1]}$

PFCs were characterized by magnetic resonance imaging or computed tomography in concordance with EUS-findings. The indications for drainage of PFCs were as follows: (1) refractory abdominal pain, (2) gastric outlet or biliary obstruction, (3) ongoing systemic illness, anorexia, and weight loss, (4) rapidly enlarging PFCs, and/or (5) infected PFCs. ${ }^{[15]}$ Patients with suspected cystic neoplasms, coagulopathy (INR >1.5) and thrombocytopenia (platelets $<50,000 / \mathrm{mm}^{3}$ ), or imaging showing that the pseudocyst wall was not in close $(>1 \mathrm{~cm})$ to the EUS probe were all excluded from the study. Data on procedural details and overall clinical course of the patient were collected from outpatient and hospital records.

\section{Description of the electrocautery-enhanced lumen-apposing metal stents}

The novel device utilized in this study, the EC-LAMS and delivery system, is a through-the-scope stent with electrocautery at the distal tip of the delivery system. 
Yoo, et al.: Electrocautery-enhanced lumen-apposing stent for pancreatic fluid collections without fluoroscopy

The stent itself has bilateral double-walled anchoring flanges designed to hold the stomach or duodenal wall in direct apposition to PFC wall. ${ }^{[16]}$ The stent is $10 \mathrm{~mm}$ in length and available in two different lumen diameters $(10 \mathrm{~mm}$ and15 $\mathrm{mm})$.

\section{Techniques}

All patients underwent procedures by two endoscopists. PFC drainage was performed using the therapeutic linear array echoendoscope (Olympus; Center Valley, PA, USA). All procedures were performed under general anesthesia, and patients were given broad-spectrum antibiotics during and after the procedure to decrease the risk of secondary infection. EUS imaging was employed to determine the optimal puncture site of the cyst (trans-gastric or transduodenal). Color Doppler was used to exclude interposed vessels at the puncture site. The PFCs were accessed from the stomach or duodenum directly with the EC-LAMS. The electrocautery tip aided passage of the catheter from the endoscope instrumentation channel into the PFC through a direct puncture of the cyst cavity without the need for wire guidance under fluoroscopy and without the need for additional dilation of the tract. The distal flange was deployed under EUS-guidance followed by positioning of this flange against the PFC wall. Deployment of the proximal flange was then performed under endoscopic guidance without fluoroscopic assistance. The selection of stent diameter $(10 \mathrm{~mm}$ or $15 \mathrm{~mm})$ was at the discretion of the endoscopist. In cases of WONs, a $15-\mathrm{mm}$ diameter was preferred because the larger diameter would allow access to the cavity for future direct endoscopic necrosectomies and better clearance of necrotic debris. The deployed stent lumen was then dilated up to $10 \mathrm{~mm}$ or $15 \mathrm{~mm}$ with a controlled radial expansion balloon to allow for optimal stent luminal expansion.

In patients with WON, endoscopic necrosectomy sessions were performed using an upper endoscope advanced through the EC-LAMS at scheduling preference of the endoscopist, usually every 3-7 days until complete resolution of the necrotic cavity as confirmed endoscopically and/or by cross-sectional imaging.

Immediate complications during or within 1 week after the procedures, such as perforation, bleeding, hypotension, or respiratory distress were carefully documented. The electronic medical records of hospital admissions and ambulatory office visits were also assessed for any delayed complications ( $<30$-day after the procedure).

Patient follow-up

Stent removal was undertaken when complete decompression of the PFC was achieved without any residual fluid component remaining. Patients were then followed at regular intervals in an outpatient setting after stent removal.

\section{Outcomes measures}

The primary outcome of this study was to evaluate the "clinical success" of drainage of PFCs through EC-LAMS without fluoroscopic guidance. "Clinical success" was defined as complete resolution of the PFC and resolution of the patient's symptoms without the need for re-intervention at 3 months following initial treatment as demonstrated on cross-sectional imaging and ambulatory follow-up.

Secondary outcomes evaluated include technical success, adverse events (AEs), procedure re-intervention, number of endoscopic procedures required for complete PFC drainage and PFC recurrence rates after stent removal. Technical success was designated as successful endoscopic placement of transmural EC-LAMS into the PFC cavity.

Immediate procedure related complications were defined as complications that occurred within 1 week after the procedure. Re-interventions were defined as the need for repeat PFC drainage as a result of stent occlusion, cyst cavity infection, or enlarging cyst size leading to symptoms.

\section{RESULTS}

Patient demographics and pancreatic fluid collection characteristics

We identified 25 patients with PFCs in whom EUS-guided transmural drainage using the EC-LAMS were performed. All PFCs developed after episodes of acute pancreatitis. The mean age of the patients was 50 years, and $56 \%$ were male $(80 \%$ white and $20 \%$ African American). The etiology of pancreatitis was gallstone (42\%), alcohol (27\%), and other causes (31\%).

The PFCs were located in the pancreatic head in 3 patients and the pancreatic body/tail in 22 patients. The mean size of the PFCs was $82 \mathrm{~mm}$ in the long axis (range of $60-170 \mathrm{~mm}$ ). Of the 25 patients, three 
Yoo, et al:: Electrocautery-enhanced lumen-apposing stent for pancreatic fluid collections without fluoroscopy

had PPs, and 22 had WONs. There was no significant difference in sex, etiology, or cyst size between patients with PP and WON.

Endoscopic ultrasound-guided pseudocyst or walled-off necrosis drainage procedure characteristics

Of the patients who underwent endoscopy for drainage of PFCs, $23(92 \%)$ had transgastric drainage and $2(8 \%)$ had trans-duodenal drainage. The cyst-gastrostomy/duodenostomy tract was dilated with a balloon in all 25 patients. Ten patients (two with PP and eight with WON) had a concomitant ERCP due to a pancreatic duct leak. Successful insertion of an EC-LAMS into the PFC cavity (technical success) was achieved in all $25(100 \%)$ patients. Of the patients who had successful placement of EC-LAMS, 23 patients had a $15 \mathrm{~mm}$ wide $\times 10 \mathrm{~mm}$ long EC-LAMS placed, and two had a $10 \mathrm{~mm} \times 10 \mathrm{~mm}$ stent positioned. Of the 22 patients with WONs, one had concomitant placement of a nasocystic tube; the nasocystic tube was irrigated with normal saline for $48-72 \mathrm{~h}$ after which it was removed. There were no procedural related complications. The median time for placement of the EC-LAMS was 10.5 (range, 7-20) $\mathrm{min}$.

Outcomes in patients with pancreatic fluid collections after successful lumen-apposing metal stent placement The mean patient follow-up period was 7.8 months with a range of 3-9 months. The median number of endoscopic sessions performed in patients with PFCs to achieve PFC resolution was 2 (range 2-6). Necrosectomy in was performed with an upper endoscope through the EC-LAMS in $71 \%$ of patients with WON stent occlusion developed in one patient who had a WON, and this patient was successfully managed through endoscopic necrosectomy without removal of the EC-LAMS.

Long-term success with endoscopic therapy of PFCs, defined as complete resolution of the PFC was achieved in 24/25 (94\%) patients. Only one patient with a WON did not achieve complete resolution. This patient was referred for surgical necrosectomy.

There was a spontaneous extrusion of one EC-LAMS into the enteral lumen after resolution of the WONs. All stents were successfully removed using a snare in all of the remaining patients after PFC resolution. In all of the cases, there were no significant AEs.

\section{DISCUSSION}

EUS-guided drainage of PFCs using novel lumen-apposing, fully covered, self-expanding metal stents has already been shown to have a high technical and long-term success rate in multiple studies. ${ }^{[13,14]}$ Conventionally, placement of LAMS has been performed with the assistance of fluoroscopy by inserting a guidewire through a needle into the cyst cavity, dilating the cystoenterostomy fistula tract using a wire-guided balloon, and finally, advancing the LAMS delivery catheter over the wire into the cyst cavity. While this method has been shown to be effective, the EC-LAMS delivery system has the potential to significantly simplify and streamline the process of deploying LAMS by allowing for a single-step procedure without the need for fluoroscopic guidance. The purpose of this study was to evaluate the clinical outcomes and technical success rates for patients who underwent a single-step deployment of EC-LAMS without fluoroscopic guidance using the EC delivery system.

Our study demonstrates that technical success and clinical outcomes of single-step deployment of EC-LAMS without the use of fluoroscopic guidance is effective and safe. Technical success was achieved in all $25(100 \%)$ patients with no procedure-related complications, and resolution of PFCs was seen in $24(96 \%)$ of patients, the one failure being in a patient with WON who was referred for surgical necrosectomy.

Similar recent studies have separately investigated either the feasibility of performing endoscopic drainage of PFCs as a streamlined single-step procedure using the EC-LAMS. Rinninella et al. evaluated 93 patients who were retrospectively studied after undergoing endoscopic treatment of PFCs using the EC-LAMS from 13 European tertiary care centers. ${ }^{[17]}$ In their study, access to the PFC was obtained directly as a single-step method using the EC-LAMS in $69(74.2 \%)$ patients. For the remaining 24 patients, access to the PFC was first obtained using a 19-gauge needle, followed by placement of a. 035-inch guidewire over which the EC-LAMS was advanced. Results of their study revealed a $98.9 \%$ technical success rate, and complete resolution of the PFC in $93.5 \%$ of cases. A report by Seicean et al. evaluated the feasibility of performing endoscopic drainage of PFCs using plastic stents without fluoroscopic guidance, though all of which were performed using a 
Yoo, et al:: Electrocautery-enhanced lumen-apposing stent for pancreatic fluid collections without fluoroscopy

guidewire. ${ }^{[18]}$ Seicean et al. found success rates comparable with previously published studies using fluoroscopy, and concluded that EUS-guided drainage of PFCs is possible, efficient, and safe without fluoroscopy in selected patients who's PFCs measure larger than $6 \mathrm{~cm}$ in diameter and have a thin wall.

The main limitation of our study is the relatively small number of patients included. In addition, the retrospective nature of the study includes inherent limitations, such as variable follow-up of patients, quality of cross-sectional imaging at different centers, and variability in the technique of the endoscopist. However, our population consisted of a heterogeneous group of patients suffering from pancreatitis of different etiologies who had considerable follow-up postprocedure.

\section{CONCLUSION}

Our study builds on prior studies by demonstrating that single-step EUS-guided drainage of PFCs without fluoroscopic guidance using novel EC-LAMS is a safe and effective endoscopic technique for the drainage of PFCs. Due to the ease of using EC-LAMS without fluoroscopic guidance, this method has the potential to significantly simplify and streamline EUS-guided management of PFCs and help in its widespread adoption as an alternative to surgery.

\section{Financial support and sponsorship}

This study was funded entirely by existing intramural funds and salary support in the respective institutions.

\section{Conflicts of interest}

There are no conflicts of interest.

\section{REFERENCES}

1. Banks PA, Bollen TL, Dervenis C, et al. Classification of acute pancreatitis-2012: Revision of the Atlanta classification and definitions by international consensus. Gut 2013;62:102-11.

2. Brun A, Agarwal N, Pitchumoni CS. Fluid collections in and around the pancreas in acute pancreatitis. J Clin Gastroenterol 2011;45:614-25.

3. Baillie J. Pancreatic pseudocysts (Part I). Gastrointest Endosc 2004;59:873-9.

4. Aghdassi A, Mayerle J, Kraft M, et al. Diagnosis and treatment of pancreatic pseudocysts in chronic pancreatitis. Pancreas 2008;36:105-12.

5. Law R, Baron TH. Endoscopic management of pancreatic pseudocysts and necrosis. Expert Rev Gastroenterol Hepatol 2015;9:167-75.

6. Baron TH, Harewood GC, Morgan DE, et al. Outcome differences after endoscopic drainage of pancreatic necrosis, acute pancreatic pseudocysts, and chronic pancreatic pseudocysts. Gastrointest Endosc 2002;56:7-17.

7. Nealon WH, Walser E. Main pancreatic ductal anatomy can direct choice of modality for treating pancreatic pseudocysts (surgery versus percutaneous drainage). Ann Surg 2002;235:751-8.

8. Akshintala VS, Saxena P, Zaheer A, et al. A comparative evaluation of outcomes of endoscopic versus percutaneous drainage for symptomatic pancreatic pseudocysts. Gastrointest Endosc 2014;79:921-8.

9. Varadarajulu S, Bang JY, Sutton BS, et al. Equal efficacy of endoscopic and surgical cystogastrostomy for pancreatic pseudocyst drainage in a randomized trial. Gastroenterology 2013;145:583-90.e1.

10. Cahen D, Rauws E, Fockens P, et al. Endoscopic drainage of pancreatic pseudocysts: Long-term outcome and procedural factors associated with safe and successful treatment. Endoscopy 2005;37:977-83.

11. Binmoeller KF, Seifert $\mathrm{H}$, Walter $\mathrm{A}$, et al. Transpapillary and transmural drainage of pancreatic pseudocysts. Gastrointest Endosc 1995;42:219-24.

12. Itoi $\mathrm{T}$, Binmoeller $\mathrm{KF}$, Shah J, et al. Clinical evaluation of a novel lumen-apposing metal stent for endosonography-guided pancreatic pseudocyst and gallbladder drainage (with videos). Gastrointest Endosc 2012;75:870-6.

13. Shah RJ, Shah JN, Waxman I, et al. Safety and efficacy of endoscopic ultrasound-guided drainage of pancreatic fluid collections with lumen-apposing covered self-expanding metal stents. Clin Gastroenterol Hepatol 2015;13:747-52.

14. Siddiqui AA, Adler DG, Nieto J, et al. EUS-guided drainage of peripancreatic fluid collections and necrosis by using a novel lumen-apposing stent: A large retrospective, multicenter U.S. experience (with videos). Gastrointest Endosc 2016;83:699-707.

15. Jacobson BC, Baron TH, Adler DG, et al. ASGE guideline: The role of endoscopy in the diagnosis and the management of cystic lesions and inflammatory fluid collections of the pancreas. Gastrointest Endosc 2005;61:363-70.

16. Singhal S, Rotman SR, Gaidhane M, et al. Pancreatic fluid collection drainage by endoscopic ultrasound: An update. Clin Endosc 2013;46:506-14.

17. Rinninella E, Kunda R, Dollhopf M, et al. EUS-guided drainage of pancreatic fluid collections using a novel lumen-apposing metal stent on an electrocautery-enhanced delivery system: A large retrospective study (with video). Gastrointest Endosc 2015;82:1039-46.

18. Seicean A, Stan-Iuga R, Badea R, et al. The safety of endoscopic ultrasonography-guided drainage of pancreatic fluid collections without fluoroscopic control: A single tertiary center experience. J Gastrointestin Liver Dis 2011;20:39-45. 